consider that a large number of patients may remain exposed to a rare but severe adverse effect.

Another case was reported to us after submission of the manuscript. In July 1993 a 63 year old man developed the Guillain-Barré syndrome after treatment for 10 days with purified monosialoganglioside for a stroke. This is the third case to be reported to us in 1993.

We gratefully acknowledge the contributions of Drs Nello Bindi (Arezzo), Riccardo Cioni (Siena), Gabriele Ciucci (Ravenna), Rosanna Fazio (Terni), Piero Meineri (Cuneo), Patrizia Perrone (Legnano), Chiara Scaccabarozzi (Lecco), Guido Tralli (Ferrara), Angelo Turchetti (Montalcino), Valter Zanardi (Belluno) and Prof Maria Giovanna Marrosu (Cagliari). We thank Professor A Del Favero, Dr M Taramelli
(Perugia), Dr E Nobile Orazio (Milano), and Dr M Guarino (Bologna) for their help. We also thank Professors C Cianchetti (Cagliari) and G Tridente (Gruppo Italiano di Neuroimmunologia) for encouraging this work.

Raschetti R, Maggini M, Popoli P. Guillain-Barré syndrome and ganglioside therapy in Italy. Lancet 1992;340:60.

2 Latov N, Koski CL, Walicke PA. Guillain-Barré syndrome and parenteral gangliosides. Lancet 1991:338:757.

3 Figueras A, Morales-Olivas FJ, Cappellà D, Palop V, Laporte JR. Bovine gangliosides and acute motor polyneuropathy. BMf 1992;305:1330-1.

4 van der Meché FGA, Schmitz PIM, and the Dutch Guillain-Barré Study Group. A randomized trial comparing intravenous immune globulin and plasma exchange in Guillain-Barré syndrome. N Engl f Med 1992;326:1123-9.

5 Granieri E, Casetta I, Govoni V, Tola MR, Paolino E, Rocca WA. Ganglioside therapy and Guillain-Barre syndrome. A historical cohort study in Ferrara, Italy, fails to demonstrate an association. Neuroepidemiology 1991;10:161-9.

(Accepted 13 fuly 1993)

\title{
Are clinicians interested in up to date reviews of effective care?
}

\section{Institute of Obstetrics and \\ Gynaecology, Royal \\ Postgraduate Medical \\ School, Queen Charlotte's \\ and Chelsea Hospital, \\ London W6 0XG \\ Sara Paterson-Brown, senior \\ registrar \\ Nicholas M Fisk, professor}

Imperial Cancer Research

Fund, London WC2A 3PX

Jeremy C Wyatt, medical

informatics manager

Correspondence to:

Miss Paterson-Brown.

$B M 7$ 1993;307:1464

\section{Sara Paterson-Brown, Jeremy C Wyatt, Nicholas M Fisk}

One of the first initiatives of the new NHS research and development strategy is the funding of the Cochrane Centre to prepare and disseminate systematic reviews of evidence based practice. This assumes that doctors will use them. Obstetrics was the first specialty to have access to such reviews with the Oxford Database of Perinatal Trials. This has been available on computer disk for four years, is updated every six months, and contains meta-analysis of randomised controlled trials in perinatal medicine. This study ascertains current awareness and use of this database.

\section{Methods and results}

We conducted a telephone survey of all 24 teaching hospitals and 74 of the 173 district hospitals in England. Of the district general hospitals in each of the 14 regional health authorities, at least a third (a minimum of four) were sampled. One of us (SP-B) interviewed either the administrative contact of the Royal College of Obstetricians and Gynaecologists (in 83 of the cases) or a suitable colleague (always a consultant; in 15 cases) with a standardised questionnaire between February and May 1993. Interviewees were aware that their responses would not be attributed to individual hospitals or doctors. All the nominated consultants were successfully contacted, and when this required multiple attempts the interviewee was not forewarned of our purpose.

Of the 24 teaching hospital consultants, 23 had heard of the Oxford database, as had 61 of the 74 from district general hospitals, but only $13(54 \%)$ and $15(20 \%)$ respectively were able to define it. It was unavailable in nine teaching hospitals-four considered it too expensive and two thought that they did not need it-

Availability and use of Oxford Database of Perinatal Trials in 98 obstetric units in England. Values are numbers (percentages) of units

\begin{tabular}{lcc}
\hline & $\begin{array}{c}\text { Teaching hospitals } \\
(\mathrm{n}=24)\end{array}$ & $\begin{array}{c}\text { District general } \\
\text { hospitals } \\
(\mathrm{n}=74)\end{array}$ \\
\hline Database unavailable & $9(38)$ & \multicolumn{2}{c}{$62(84)$} \\
Database available and used for: & $15(62)$ & \multicolumn{2}{c}{$12(16)$} \\
$\quad$ Planning research & 8 & 1 \\
Finding references & 11 & 6 \\
Education & 13 & 7 \\
Designing protocols & 10 & 8 \\
Specific patient problems & 2 & 6 \\
Unknown & 1 & 2 \\
\hline
\end{tabular}

and in 62 district general hospitals-reasons given included the hospital not being computerised (in seven cases) and not needing it (10). In the units with the database, however, it was used by all grades of doctor between once a week and once a month.

\section{Comment}

This survey shows that systematic overviews of evidence based practice are not currently well disseminated, with $72 \%$ of the obstetric units surveyed not having access to a copy of the database of obstetric trials. Despite publicity and support for such overviews in obstetrics ${ }^{1-3}$ lack of awareness was shown to be an important factor, as $14 \%$ of obstetric consultants had never heard of the database and only $29 \%$ could define it. We also questioned interviewees on their awareness and possession of the now outdated textbook version (Effective Care in Pregnancy and Childbirth) advocated in the maternity services report $t^{4}$ and will report the findings elsewhere.

Among the reasons given for not having the database, seven obstetric units in district general hospitals did not have access to a computer, which is surprising. Another reason given was one of expense: in 1989 the database cost about $£ 600$, in 1991 about $£ 250$, but now, produced by The Cochrane Collaboration, it costs $£ 56.40$ a year for a personal copy or $£ 98.70$ for an institution. The justification given for some units not having the database revealed entrenched attitudes: "Obstetrics is an art not a science"; "We are a teaching hospital so we don't need to know what everyone else does"; and "We have been able to avoid writing protocols to date."

Obstetricians have been singled out for having unscientific and inconsistent practices. ${ }^{4}$ Clinical protocols are becoming more popular, but without access to the database incorrect management policies will remain widespread. An example of this is a recent article in the $B M F$ which contains advice on the management of post-term pregnancy that is in contradiction to the database. ${ }^{5} \mathrm{Up}$ to date reviews of effective care may have medicolegal relevance in the future.

Despite its obvious benefits, the database is neither widely disseminated nor widely used. These problems should be noted by other branches of medicine now trying to disseminate systematic reviews of effective care.

1 Paintin DB. Effective care in pregnancy and childbirth. Br $f$ Obstet Gynaecol 1990;97:967-9.

2 Fisk NM. Software review. Early Hum Dev 1990;24:181-3.

3 Evidence-Based Medicine Working Group. Evidence based medicine: a new approach to teaching the practice of medicine. $¥ A M A$ 1992;268:2420-5

4 House of Commons Health Committee. Maternity services. London: HMSO, 1992.

5 Cardozo L. Is routine induction of labour at term ever justified? $B M \mathcal{F}$ 1993;306:840-1.

(Accepted 10 September 1993) 